\title{
Rehabilitation Outcomes and Infection Control in the Early Stage of the COVID-19 Pandemic
}

\author{
Yukimasa Igawa ${ }^{1 *}$, Takahiro Sugimoto ${ }^{2}$ \\ ${ }^{I}$ Department of Internal Medicine and Rehabilitation, Aizen Hospital, Sapporo, 005-0813, Japan. \\ ${ }^{2}$ Department of Rehabilitation, Aizen Hospital, Sapporo, 005-0813, Japan.
}

Received 13 March 2021; Revised 08 April 2021; Accepted 24 April 2021; Published 27 April 2021

\begin{abstract}
Introduction: Understanding how infection control measures were devised and the outcomes of rehabilitation in the early stage of an emerging infectious disease pandemic is important. This study aimed to investigate the impact of the coronavirus disease 2019 (COVID-19) pandemic on inpatient rehabilitation, and to determine the effectiveness of the original infection control measures implemented for the rehabilitation team. Material and Methods: In this single-center, retrospective, observational study, we calculated multiple rehabilitation indices of patients discharged from our rehabilitation ward between February 28 and May 25, 2020 when Hokkaido was initially affected by COVID-19, and compared them with those calculated during the same period in 2019. Fisher's exact test and the Mann-Whitney U test were used for statistical analysis. We also verified the impact of implementing the original infection control measures for the rehabilitation team on preventing nosocomial infections. Results: A total of 93 patients (47 of 2020 group, 46 of 2019 group) were included. The median age was 87 and 88 years, respectively, with no differences in age, sex, and main disease between the groups. Training time per day in the ward in 2020 was significantly lower than that in 2019 (p = 0.013). No significant differences were found in the qualitative evaluation indices of Functional Independence Measure (FIM) score at admission, FIM gain, length of ward stay, FIM efficiency, and rate of discharge to home. None of the patients or staff members had confirmed COVID-19 during the study period. Conclusions: Early COVID-19 pandemic affected the quantitative index for inpatient rehabilitation but not the qualitative indices. No symptomatic nosocomial COVID-19 infections were observed with our infection control measures.
\end{abstract}

Keywords: COVID-19; Rehabilitation Hospital; Outcome Assessment; Nosocomial Infection.

\section{Introduction}

In December 2019, an emerging infectious disease caused by severe acute respiratory syndrome coronavirus 2 (SARS-CoV-2) was reported from China [1], and the World Health Organization declared coronavirus disease 2019 (COVID-19) a global pandemic on March 11, 2020 [2]. In Japan, the first case of COVID-19 was reported in January 2020 [3]. Since then, the number of reported cases has continued to increase and Hokkaido has become the most heavily impacted area in Japan. Taking the situation very seriously, the Hokkaido Government issued a "Declaration of State of Emergency in Hokkaido" on February 28, 2020 [4]. With a decrease in the number of reported cases, Hokkaido's declaration of a state of emergency was then lifted on March 19. However, the number of reported infections increased again across the country, including the Hokkaido area, in early April, and the prime minister of Japan issued a nationwide declaration of a state of emergency on April 16. This increase was considered to be the

* Corresponding author: pinoharu2@gmail.com

doi) http://dx.doi.org/10.28991/SciMedJ-2021-03-SI-6

$>$ This is an open access article under the CC-BY license (https://creativecommons.org/licenses/by/4.0/).

(C) Authors retain all copyrights. 
second wave of COVID-19 in Hokkaido, which had not been seen nationally or globally. The declared state of emergency in Hokkaido continued until May 25, when it was lifted nationwide [5]. The early spread of the COVID-19 epidemic and the second wave of outbreaks in Hokkaido had a great impact on medical institutions and their medical staff, as well as hospitalized patients and families. Several facilities with nosocomial COVID-19 infections were reported.

Rehabilitation medicine inevitably involves close contact with patients and provides many opportunities to meet with patients and their family members to assist with hospital discharge. We hypothesized that the original infection control measures implemented for the rehabilitation team would enable both prevention of nosocomial infections and allow usual outcomes of rehabilitation medicine, even in the setting of extreme shortage of medical resources. To test this hypothesis, we investigated the impact of COVID-19 pandemic on inpatient rehabilitation and the effectiveness of the original infection measures implemented for the rehabilitation team.

\section{Material and Methods}

\subsection{Study Design}

Single-center, retrospective, observational study.

\subsection{Study Facility}

Rehabilitation ward of a hospital in the early epidemic area of Japan, which did not treat patients with apparent COVID-19 or SARS-CoV-2 infection.

\subsection{Subjects and Evaluation Items}

The detailed evaluation indices obtained from discharged patients between February 28, 2020, and May 25, 2020, were compared with those obtained for the same period in 2019. The study period in 2020 started from the declaration of Hokkaido's state of emergency and ended with the lifting of the nationwide state of emergency. Patients who were transferred to another hospital or ward because of worsening condition or adverse events and those that expired during the study period were excluded. We used the Functional Independence Measure (FIM) to measure changes in functional ability. The tool consists of 18 items, each of which is graded on a 7-point ordinal scale [6]. The evaluation items were age, sex, main disease name, FIM score at admission, gain of FIM, length of ward stay, FIM efficiency, rate of discharge to home, and training time per day. Discharge to home was defined as discharge to the patient's own residence or residential facility.

\subsection{Statistical Analyses}

The Fisher exact test was used to analyze sex, main disease, and rate of discharge to home of the subjects. The Mann-Whitney U test was used to analyze the subjects' age, FIM score at admission, FIM gain, FIM efficiency, and training time. The level of significance was set at $\mathrm{p}<0.05$, and the statistical software program used was EZR [7].

\subsection{Nosocomial Infection Control}

The infection control committee in the hospital formulated nosocomial infection control measures based on the knowledge provided by related academic societies and public institutions. The nature of rehabilitation medicine was also taken into consideration, with rehabilitation staff meetings in the hospital to consider the formulation of the original infection control measures for the rehabilitation team. Table 1 shows the infection control measures for the rehabilitation team assembled by the staff. The handling of suspected cases and polymerase chain reaction (PCR) testing for COVID-19 was supposed to be carried out by a local governmental healthcare center upon request from attending clinicians, based on the guidelines of the central government [8].

\section{Results}

\subsection{Evaluation Indices Measured in Discharged Patients}

Fifty-four patients in 2020 and 58 in 2019 were discharged from the rehabilitation ward. Forty-seven study subjects in 2020 and 46 in 2019 ultimately met the inclusion criteria. Table 2 shows the patients demographic information, their main diagnoses, and their evaluation index data. No significant differences in demographics and diagnoses were observed between the two groups $(\mathrm{p}>0.05)$. No significant differences were found in FIM score at admission, FIM gain, length of ward stay, FIM efficiency, or rate of discharge to home ( $p>0.05)$. Only the training time per day showed a significant decrease $(\mathrm{p}=0.013)$ in the 2020 group compared with that in the 2019 group. Figure 1 shows a dot plot of the distribution of the training time per patient per day. The distribution of dots in the 2020 group was moved down compared with that in the 2019 group. 
Table 1. Infection control measures for the rehabilitation team

\section{Common}

Team members should work in a single ward and should not work in other departments.

PPE* should be used in accordance with the supply and availability.

\section{Droplets}

All circumstances, including conferences, must be designed to avoid the $3 \mathrm{Cs} \uparrow$.

Keep appropriate physical distance from patients or staff during functional training.

Wear both face mask and face shield during functional training.

Avoid face to face conversations with patients as much as possible.

Patients should be asked to wear a face mask if the supply is sufficient.

\section{Contact}

Functional training should be provided around a patient's bedside or in-ward.

Avoid careless touching of environment around patients' beds.

Avoid sharing training instruments or equipment.

Disinfect instruments or equipment after each use if sharing is needed.

Frequently disinfect PCs and other devices shared by staff members.

Note: Standard precautions and staff quarantine are to be managed by the infection control committee in the hospital.

PPE*: Personal protective equipment

The 3Cs $\uparrow$ : Closed spaces with poor ventilation, Crowded places with many people nearby, and Close-

contact settings such as close-range conversations

Table 2. Basic information and evaluation indices for discharged patients

\begin{tabular}{|c|c|c|c|}
\hline & 2020 & 2019 & $p^{q /}$ \\
\hline $\mathrm{n}$ & 47 & 46 & \\
\hline Age $[\text { years }]^{\ddagger}$ & $87(80.5-91)$ & $88(82.3-92.8)$ & 0.249 \\
\hline Male female & $13 \quad 34$ & $17 \quad 29$ & 0.38 \\
\hline CNS/Orthopedics/other ${ }^{\S}$ & $11 / 35 / 1$ & $13 / 33 / 0$ & 0.726 \\
\hline${ }^{\| F I M}$ at admission ${ }^{\ddagger}$ & $61(49.5-80.5)$ & $64(46.5-79.5)$ & 0.982 \\
\hline${ }$ FIM gain ${ }^{\ddagger}$ & $26(17.5-35)$ & $26(10.3-34.8)$ & 0.569 \\
\hline Length of ward stay & $63(47-96.5)$ & $69.5(49.3-82.8)$ & 0.833 \\
\hline${ }^{\| F I M}$ efficiency ${ }^{\ddagger}$ & $0.47(0.2-0.64)$ & $0.44(0.19-0.62)$ & 0.429 \\
\hline Rate of discharge to home & $80.9 \%$ & $89.1 \%$ & 0.762 \\
\hline Training time per day $[\mathrm{min}]^{\ddagger}$ & $144.6(122.3-155.4)$ & $155.7(138.9-165.4)$ & 0.013 \\
\hline
\end{tabular}

Note: $\ddagger$ Numbers are given as median (interquartile range). §Central nervous system: cerebrovascular disease or spinal cord disease. Orthopedics: bone fracture or ligament injury or disc herniation. Other: disuse syndrome. |FIM: Functional independence measure. IThe $\mathrm{P}$ values for sex, main disease, and rate of discharge to home were calculated using the Fisher exact test. Age, FIM at admission, FIM gain, FIM efficiency, and training time were calculated using the Mann-Whitney U test.

\subsection{Nosocomial Infections}

No patients or staff members in the hospital had confirmed or suspected cases of COVID-19 during the study period. None of the patients or staff members were eligible for PCR test based on case definitions in the guidelines, including the criteria for consultation and reporting to the local governmental healthcare center. 


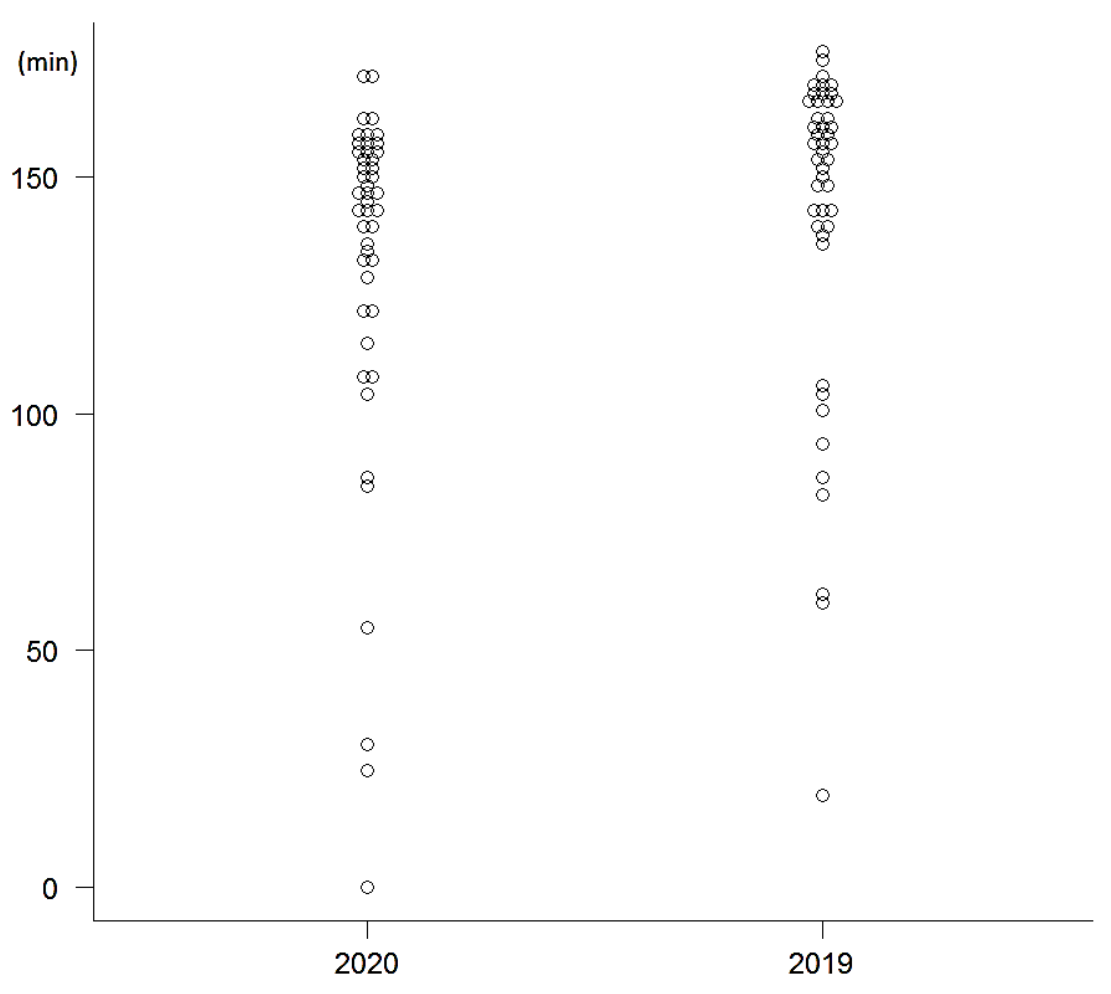

Figure 1. Training time of discharged patients. The distribution of training time of a discharged patient per day is shown. Statistical details are described in Table 2.

\section{Discussion}

The aim of rehabilitation is to maximize function and minimize the limitations of activity and restriction in participation resulting from an underlying impairment or disease $[9,10]$. Rehabilitation outcomes have often been measured with evaluation indices based on FIM, which were designed to quantify the degree of disability [6, 11]. Adding length of ward stay, rate of discharge to home, and training time as the evaluation indices in this study allowed a more detailed and multi-layered assessment. FIM gain is calculated by subtracting the FIM score at admission from the FIM score at discharge, and FIM efficiency is calculated by dividing the FIM gain by the number of days in the ward, all of which are indicators of the quality of inpatient rehabilitation [12].

If the pandemic had an impact on the quality of inpatient care, some changes in FIM-based indices and hospital length of stay should have been observed since COVID-19 is not simply a respiratory infection, but rather affects society and medicine at large. However, the 2020 group had the same length of ward stay, unchanged FIM gain, and increased FIM efficiency. The indices obtained from the discharged patients during the study period showed no significant differences, except for the training time, between the 2020 and 2019 groups. The possible reasons for the statistically significant decrease in training time include a thorough staff quarantine under the state of emergency due to the COVID-19 outbreak, where rehabilitation therapists with the slightest change in health conditions such as the emergence of cold symptoms were instructed to stay home. Most countries, including Japan, provide guidelines for identifying healthcare workers with suspected COVID-19, which can restrict their attendance based on selfassessments including fever [13]. These staffing limitations might have affected the capability to provide training time during the study period. The sudden suspension of rehabilitation therapists from work appears to have had the effect of pushing down the distribution of dots per patient per day in 2020 (Figure 1). However, it is not possible to determine from this study whether the statistically significant reduction in training time had any clinical significance. Even so, the fact that COVID-19 pandemic had a significant impact on training time in inpatient rehabilitation indicates that in the uncertain future of this pandemic, telemedicine and self-training must be enhanced to complement the reduced training time [14].

None of the patients or staff members were observed with clinically apparent nosocomial COVID-19 during the study period. Nosocomial infection control is a top priority during the COVID-19 outbreak. Centers for Disease Control and Prevention published successive updates to guidelines intended for medical institutions [15]. However, there was little mention of rehabilitation medicine or facilities in the guidelines. This had led the physicians and administrators involved to develop their own infection control measures. Infection control in rehabilitation medicine is complicated by the considerable amount of contact with patients, close physical proximity, frequency of conversations, 
frequent production of droplets during speech therapy training or eating and swallowing training, frequent crowding among staff at conferences, and sharing of instruments and equipment. Estimates of the route of infection with COVID-19 have reportedly identified that approximately $10 \%$ of infections are contracted through environments such as high-frequency contact surfaces [16]. In addition to the recommended infection control measures for COVID-19 from most of the public institutes, additional measures addressing contact with equipment are required in rehabilitation medicine, where the frequency of such contact is high. Although the possibility of fomite transmission in the community is considered low, its occurrence in healthcare settings should not be underestimated [17].

The use of surgical masks and face shields by healthcare professionals has previously been recommended and reviewed, mainly with the purpose of protecting healthcare professionals' own body from exposure to sources of infection [18]. In the case of COVID-19, however, non-first-line healthcare workers had a higher infection rate than first-line healthcare workers, which differed from the observations in the previous viral disease epidemics [19]. In the clinical setting of rehabilitation medicine, young medical staff providing care to older patients is quite common. The older the patient and the more chronic diseases they have, the more vulnerable they are to the virus [20], and therefore, protecting hospitalized older adults from staff-mediated infections has been an urgent issue [21]. The concept of source control was proposed to address this issue, and global advocation for universal face masking had emerged [22]. For face shields, verification of the assumed influenza infection cases that were reported revealed a $96 \%$ decrease in the risk of droplet inhalation [23]. A pilot study reported that universal face shield use was associated with marked reduction in infections among healthcare personnel and hospitalized patients [24]. Although the effectiveness of using face shields in COVID-19 remains unclear, it is common sense to wear them for protection. Even in the context of an extreme shortage of Personal Protective Equipment (PPE), face shields can be handmade from commonplace materials, making them an option for universal use, as well as for use by non-first-line medical care workers [25], including those in rehabilitation medicine.

The infection control measures implemented based on these findings have allowed us to continue our inpatient care without a single case of infection in an inpatient member throughout the year 2020. However, strategic testing of asymptomatic individuals to prevent transmission is key until safe and effective vaccines are widely available [26]. In the epicenter of the global pandemic, such as the United States and Europe, some rehabilitation wards were converted to COVID-19 treatment wards due to an outbreak of the infection in the surrounding areas, interrupting the usual medical care for rehabilitation [27, 28]. In contrast, the impact on inpatient rehabilitation in Hokkaido, Japan, in the early stages of the pandemic as shown in this study was limited. We were able to achieve good patient outcomes even with the reduced hours of rehabilitation training that came with COVID-19 restrictions. However, these outcomes achieved with the staffing constraints resulted in longer hours and physical and mental burnout for medical personnel. Our observations correspond closely with the reports from other epidemic areas of the world and need to be addressed in a world with COVID-19 [29].

This study has some important limitations. First, the prevalence and impact of COVID-19 were dependent on regionality and national insurance systems. Second, this study was a retrospective observational design and was conducted at a single facility in the early epidemic area of Japan. Third, this emerging infectious disease is expected to last long. The results of this study reflect the situation during the very early stages of the pandemic, when PPEs were extremely scarce, and are descriptive of the response process and its results. The response to the upcoming waves of COVID-19 might improve significantly as the issues of low PPE supply and COVID-19 management strategy have improved over time.

\section{Conclusion}

We demonstrated that the early-stage of the COVID-19 pandemic in Hokkaido, Japan, did not negatively affect the qualitative outcomes of inpatient rehabilitation, even in the setting of extreme shortage of medical resources. The staffing constraints resulting in physical and/or mental burnout of medical personnel remains a problem to be resolved. This study suggests that it is possible to provide conventional rehabilitation outcomes with minimal negative influences during the pandemic if appropriate infection control measures are implemented. In the COVID-19 era, safe and effective vaccines, and Webinar medical systems should be more widely used globally, including in developing countries.

\section{Declarations}

\subsection{Author Contributions}

Y.I. wrote the first draft of the manuscript; T.S. analyzed and interpreted the data. All authors have read and agreed to the published version of the manuscript.

\subsection{Funding}

The authors received no financial support for the research, authorship, and/or publication of this article. 


\subsection{Acknowledgements}

We gratefully acknowledge all members of the infection control team in Aizen Hospital for their dedicated work under the pandemic situation of COVID-19. The authors also thank Dr. Shuji Dohi, head of the hospital, for his kind support and advice.

\subsection{Ethical Approval}

This was a retrospective, observational study, conducted in accordance with the tenets of the Declaration of Helsinki, guaranteeing complete anonymity. This study was approved by the Ethics Committee of Aizen Hospital (Permission number: 2020-003).

\subsection{Data Availability Statement}

The data presented in this study are available on request from the corresponding author.

\subsection{Conflict of Interest}

The authors declare that they have no known competing financial interests or personal relationships that could have appeared to influence the work reported in this paper.

\section{References}

[1] Hui, D. S., Azhar, E. I., Madani, T. A., Ntoumi, F., Kock, R., Dar, O., ... \& Petersen, E. (2020). The continuing 2019-nCoV epidemic threat of novel coronaviruses to global health-The latest 2019 novel coronavirus outbreak in Wuhan, China. International journal of infectious diseases, 91, 264-266. doi:10.1016/j.ijid.2020.01.009.

[2] World Health Organization: WHO Director-General's opening remarks at the media briefing on COVID-19. 11 March, 2020. Available online: https://www.who.int/director-general/speeches/detail/who-director-general-s-opening-remarks-at-the-mediabriefing-on-covid-19---11-march-2020 (accessed on March 2020).

[3] Yuki Furuse, Yura K Ko, Mayuko Saito, Yugo Shobugawa, Kazuaki Jindai, Tomoya Saito, Hiroshi Nishiura, Tomimasa Sunagawa, Motoi Suzuki, National Task Force for COVID-19 Outbreak in Japan. Epidemiology of COVID-19 Outbreak in Japan, January-March 2020. Jpn J Infect Dis 2020. doi:10.7883/yoken.JJID.2020.271.

[4] Shunji Edagawa, Fumiko Kobayashi, Fumihiro Kodama, Masayuki Takada, Yuki Itagaki, Akira Kodate, Keisuke Bando, Keisuke Sakurai, Akio Endo, Hisako Sageshima, Atsushi Nagasaka. Epidemiological features after emergency declaration in Hokkaido and report of 15 cases of COVID-19 including 3 cases requiring mechanical ventilation. Global Health \& Medicine 2020; 2, 112-117. doi:10.35772/ghm.2020.01024.

[5] Mun-Keat Looi. Covid-19: Japan ends state of emergency but warns of "new normal". BMJ 2020; 369: m2100. doi:10.1136/bmj.m2100.

[6] Linacre, J. M., Heinemann, A. W., Wright, B. D., Granger, C. V., \& Hamilton, B. B. (1994). The structure and stability of the Functional Independence Measure. Archives of physical medicine and rehabilitation, 75(2), 127-132. doi:10.1016/00039993(94)90384-0.

[7] Kanda Y. Investigation of the freely-available easy-to-use software "EZR" (Easy R) for medical statistics. Bone Marrow Transplant 2013:48,452-458. doi:10.1038/bmt.2012.244.

[8] Information for Public. Q\&A on Coronavirus Disease 2019 (COVID-19). Ministry of Health, Labor and Welfare.2020. Available online: https://www.mhlw.go.jp/stf/seisakunitsuite/bunya/kenkou_iryou/dengue_fever_qa_00014.html (accessed on March 2020).

[9] WHO Expert Committee on Disability Prevention and Rehabilitation \& World Health Organization. (1981). Disability prevention and rehabilitation: report of the WHO Expert Committee on Disability Prevention and Rehabilitation (meeting held in Geneva from 17 to 23 February 1981). World Health Organization. Available online: https://apps.who.int/iris/handle/10665 140896 (accessed on December 2020).

[10] S Bachmann, C Finger, A Huss, M Egger, AE Stuck, and KM Clough-Gorr. Inpatient rehabilitation specifically designed for geriatric patients: systematic review and meta-analysis of randomised controlled trials. BMJ 2010;340:c1718. doi:10.1136/bmj.c1718.

[11] Ribeiro DKMN, Lenardt MH, Lourenço TM, Betiolli SE, Seima MD, Guimarães CA. The use of the functional independence measure in elderly. Rev Gaúcha Enferm 2017; 38(4): e66496. doi:10.1590/1983-1447.2017.04.66496.

[12] M. Tokunaga, S. Mita, Keiichi Tashiro, M. Yamaga, Y. Hashimoto, R. Nakanishi, H. Yamanaga. Methods for comparing Functional Independence Measure improvement degree for stroke patients between rehabilitation hospitals. Int J Phys Med Rehabil 2017;5:2. doi:10.4172/2329-9096.1000394. 
[13] Centers for Disease Control and Prevention. Operational Considerations for the Identification of Healthcare Workers and Inpatients with Suspected COVID-19 in non-US Healthcare Settings. Available online: https://www.cdc.gov/coronavirus/ 2019-ncov/hcp/non-us-settings/guidance-identify-hcw-patients.html (accessed on May 2020).

[14] Turolla, A., Rossettini, G., Viceconti, A., Palese, A., \& Geri, T. (2020). Musculoskeletal physical therapy during the COVID19 pandemic: is telerehabilitation the answer?. Physical therapy, 100(8), 1260-1264. doi:10.1093/ptj/pzaa093.

[15] Centers for Disease Control and Prevention. (2020). Infection Control Guidance for Healthcare Professionals about Coronavirus (COVID-19). Available online: https://www.cdc.gov/coronavirus/2019-ncov/hcp/infection-control.html (accessed on March 2020).

[16 Ferretti, L., Wymant, C., Kendall, M., Zhao, L., Nurtay, A., Abeler-Dörner, L., ... \& Fraser, C. (2020). Quantifying SARSCoV-2 transmission suggests epidemic control with digital contact tracing. Science, 368(6491). doi:10.1126/science.abb6936.

[17] Leung, N. H. (2021). Transmissibility and transmission of respiratory viruses. Nature Reviews Microbiology, 1-18. doi:10.1038/s41579-021-00535-6.

[18] Centers for Disease Control and Prevention. Protecting Healthcare Personnel. Available online: https://www.cdc.gov/hai/prevent/ppe.html (accessed on May 2020).

[19] Xiaoquan Lai, Minghuan Wang, Chuan Qin, Li Tan, Lusen Ran, Daiqi Chen, Han Zhang, Ke Shang, Chen Xia, Shaokang Wang, Shabei Xu, Wei Wang. Coronavirus Disease 2019 (COVID-2019) Infection among Health Care Workers and Implications for Prevention Measures in a Tertiary Hospital in Wuhan, China. JAMA Netw Open. 2020 May 1; 3(5):e209666. doi:10.1001/jamanetworkopen.2020.9666.

[20] Christopher M Petrilli, Simon A Jones, Jie Yang, Harish Rajagopalan, Luke O'Donnell, Yelena Chernyak, Katie A Tobin, Robert J Cerfolio, Fritz Francois, Leora I Horwitz. Factors associated with hospital admission and critical illness among 5279 people with coronavirus disease 2019 in New York City: prospective cohort study. BMJ 2020; 369:m1966. doi:10.1136/bmj.m1966.

[21] Temet M McMichael, Dustin W Currie, Shauna Clark, Sargis Pogosjans, Meagan Kay, Noah G. Schwartz, James Lewis, Atar Baer, Vance Kawakami, Margaret D. Lukoff, Jessica Ferro, Claire Brostrom-Smith, Thomas D. Rea, Michael R. Sayre, Francis X. Riedo, Denny Russell, Brian Hiatt, Patricia Montgomery, Agam K. Rao, Eric J. Chow, Farrell Tobolowsky, Michael J. Hughes, Ana C. Bardossy, Lisa P. Oakley, Jesica R. Jacobs, Nimalie D. Stone, Sujan C. Reddy, John A. Jernigan, Margaret A. Honein, Thomas A. Clark, Jeffrey S. Duchin, for the Public Health-Seattle and King County, EvergreenHealth, and CDC COVID-19 Investigation Team. Epidemiology of Covid-19 in a Long-Term Care Facility in King County, Washington. N Engl J Med 2020 May 21; 382(21):2005-2011. doi:10.1056/nejmoa2005412.

[22] Klompas M, Morris CA, Sinclair J, Pearson M, Shenoy ES. Universal masking in hospitals in the COVID-19 Era. N Engl J Med 2020;382:e63. doi:10.1056/nejmp2006372.

[23] Lindsley WG, Noti JD, Blachere FM, Szalajda JV, Beezhold DH. Efficacy of face shields against cough aerosol droplets from a cough simulator. J Occup Environ Hyg 2014; 11(8):509-518. doi:10.1080/15459624.2013.877591.

[24] Mayar Al Mohajer, Kristen M. Panthagani, Todd Lasco, Bradley Lembcke, Vagish Hemmige. Association between universal face shield in a quaternary care center and reduction of SARS-COV2 infections among healthcare personnel and hospitalized patients. Int J Infect Dis. 2021 Apr; 105: 252-255. doi:10.1016/j.ijid.2021.02.060.

[25] Perencevich EN, Diekema DJ, Edmond MB. Moving personal protective equipment into the community: face shields and containment of COVID-19. JAMA 2020;323(22):2252-2253. doi:10.1001/jama.2020.7477.

[26] Michael A Johansson, Talia M Quandelacy, Sarah Kada, Pragati Venkata Prasad, Molly Steele, John T Brooks, Rachel B Slayton, Matthew Biggerstaff, Jay C Butler. SARS-CoV-2 Transmission From People Without COVID-19 Symptoms. JAMA Netw Open. 2021 Jan 4;4(1):e2035057. doi:10.1001/jamanetworkopen.2020.35057.

[27] Escalon, M. X., \& Herrera, J. (2020). Adapting to the Coronavirus Disease 2019 Pandemic in New York City. American Journal of Physical Medicine \& Rehabilitation, 99(6), 453-458. doi:10.1097/phm.0000000000001451.

[28] Paolo Boldrini, Carlotte Kiekens, Stefano Bargellesi, Rodolfo Brianti, Silvia Galeri, Lucia Lucca. First impact on services and their preparation. "Instant paper from the field" on rehabilitation answers to the Covid-19 emergency. Eur J Phys Rehabil Med 2020 Jun;56(3):319-322. doi:10.23736/s1973-9087.20.06303-0.

[29] Qian Liu, Dan Luo, Joan E Haase, Qiaohong Guo, Xiao Qin Wang, Shuo Liu, Lin Xia, Zhongchun Liu, Jiong Yang, Bing Xiang Yang. The experiences of health-care providers during the COVID-19 crisis in China: a qualitative study. Lancet Glob Health 2020 Jun;8(6):e790-e798. doi:10.1016/S2214-109X(20)30204-7. 